\title{
Nanoscale Infrared Spectroscopy: A non-Destructive Probe of Formation History in Extraterrestrial Samples
}

\author{
A. S. McLeod ${ }^{1}$, G. Dominguez ${ }^{2,3}$, Z. Gainsforth ${ }^{4}$, P. Kelly ${ }^{1}$, F. Keilmann ${ }^{5}$, A. Westphal ${ }^{4}$, M. Thiemens ${ }^{2}$, \\ and D. N. Basov ${ }^{1}$ \\ ${ }^{1}$ University of California, San Diego, Department of Physics, La Jolla, CA 92093, USA \\ ${ }^{2}$ University of California, San Diego, Dept. of Chemistry and Biochemistry, La Jolla, CA 92093, USA \\ ${ }^{3}$ California State University, San Marcos, Department of Physics, San Marcos, CA 92096-0001, USA \\ ${ }^{4}$ Space Sciences Laboratory, University of California, Berkeley, Berkeley, CA 94720, USA \\ ${ }^{5}$ LASNIX, Sonnenweg 32, 82152 Berg, Germany
}

Advances in the spatial resolution of modern analytical techniques have tremendously augmented the scientific insight gained from analysis of natural samples. Yet, while techniques for the elemental and structural characterization of samples have achieved sub-nanometer spatial resolution, infrared spectral mapping of geochemical samples at vibrational "fingerprint" wavelengths has remained restricted to spatial scales larger than 10 microns. Nevertheless, infrared spectroscopy remains an invaluable contactless probe of chemical structure, details of which offer clues to the formation history of minerals. Here we report on successful implementation of non-destructive infrared near-field imaging, spectroscopy and analysis techniques capable of sub-micron scale mineral identification within natural samples, including a chondrule from the Murchison meteorite (a CM2 chondrite) and a dust grain (Iris) returned from comet 81P/Wild 2 by NASA's Stardust mission $[1,3,4]$. This work inaugurates a new era of infrared nanoscopy applied to small and invaluable extraterrestrial samples.

Recent years have seen development of Apertureless Near-field Scanning Optical Microscopy (ANSOM) utilizing a metallic atomic force microscope (AFM) probe as an optical antenna to focus incident electromagnetic radiation to areas smaller than the wavelength of incident light. By incorporating a broadband mid-infrared (MIR) laser and spectroscopic interferometer into an ANSOM, the investigators have achieved $20 \mathrm{~nm}$-resolved Fourier Transform Infrared (nanoFTIR) spectroscopy capable of fingerprinting geochemical materials by their vibrational signatures in a fashion analogous to conventional infrared absorption spectroscopies [2]. Here we report on the first application of infrared ANSOM and nanoFTIR with a metallic near-field probe to identify and distinguish silicate minerals within extraterrestrial samples with sub-micron lateral resolution. A sophisticated model of the optical interactions associated with ANSOM permit unambiguous interpretation of nanoFTIR spectra.

Correlative scanning electron microscopy (SEM), energy dispersive X-ray spectroscopy (EDS), and transmission electron microscopy are applied on these samples to benchmark and verify material characterizations provided by nanoFTIR. Besides a surface polish providing sub-micron smoothness, nanoFTIR is found to require no specialized sample preparation, providing a level of convenience heretofore unavailable with former techniques, while conferring competitive resolution and chemical sensitivity. We demonstrate that nano-scale infrared spectroscopy can 1) resolve cation concentration gradients associated with rapid cooling at the level of a few percent, 2) identify sub-micron intergrowths at the confluence of distinct pyroxenes, providing evidence for shock within a chondrule, and 3) directly confirm the proximity of crystalline and amorphous silicates in the Iris cometary grain, reinforcing the unexpected conclusion that chondrites and comets likely shared a common formation process within the presolar nebula $[1,3,4]$. 

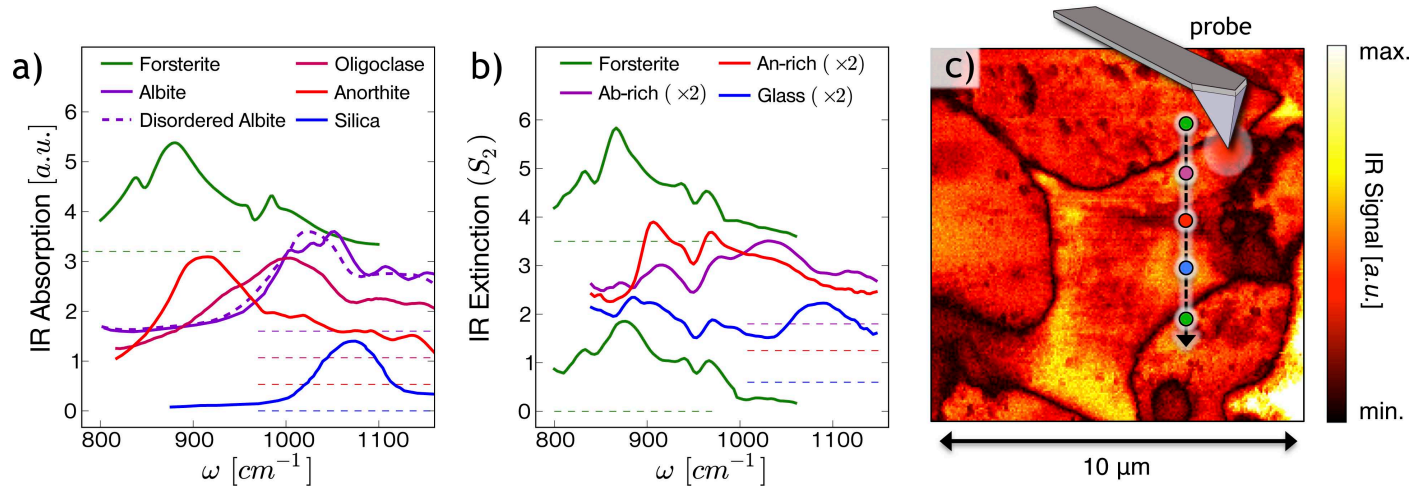

Fig. 1 (a) Literature IR absorption spectra from forsterite, various plagioclase feldspars, and amorphous silica. Oligoclase and anorthite spectra are from powdered samples, silica from a thin film, and all others from bulk (crystalline) samples. (b) Region-averaged IR extinction spectra extracted from a nanoFTIR line-scan through a cometary dust grain returned from comet 81P / Wild 2. Extracted forsterite spectra agree well with reported absorption spectra, whereas central plagioclase regions exhibit albite (Ab)-rich, anorthite (An)- rich, and glassy signatures. Sharpness of anorthite and oligoclase nanoFTIR peaks imply crystalline material, whereas reported spectra for powders (a) display added broadening. The broad nanoFTIR albite peak is consistent with strong Al-Si disorder, as from rapid quenching from a melt. (c) Infrared near-field image collected from the line-scan area, with positions of nanoFTIR spectra indicated by color.
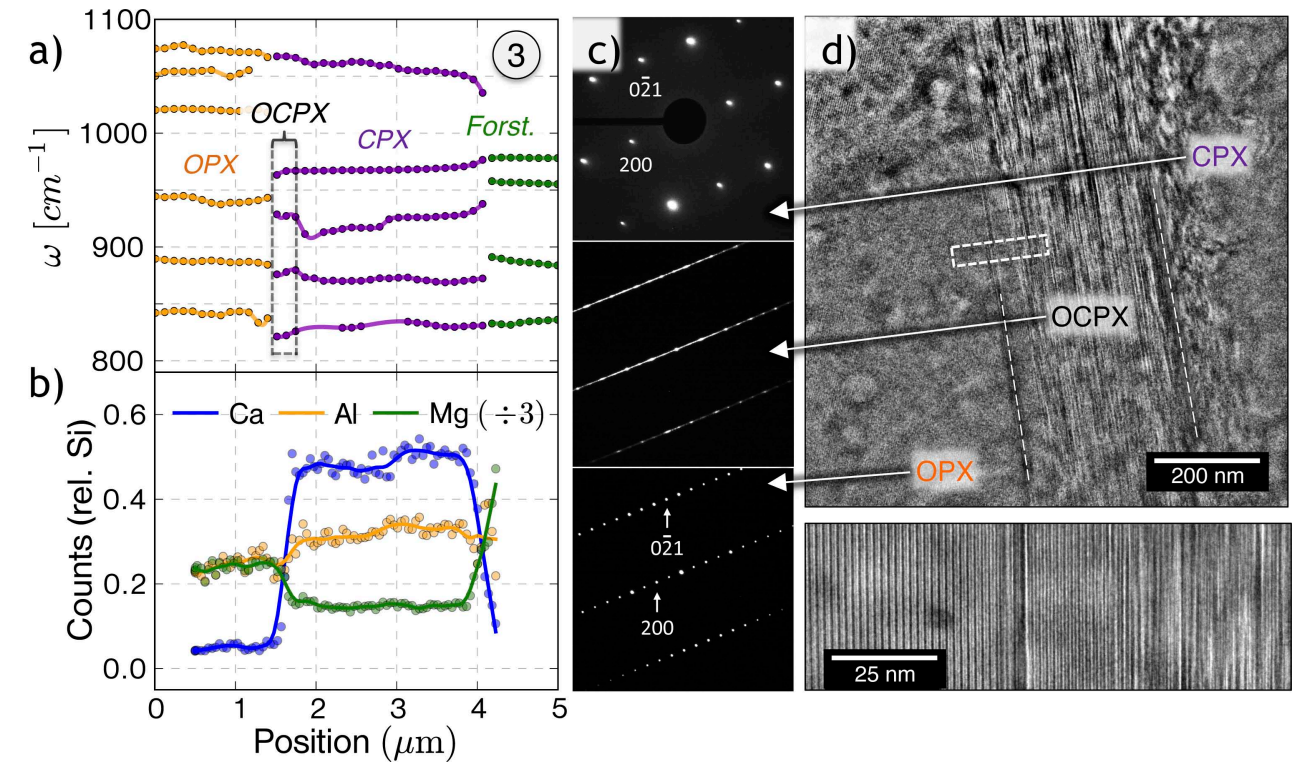

Fig. 2 (a) Vibrational frequencies versus position resolved by nanoFTIR at the nano-scale through a chondrule of the Murchison meteorite. Forsterite (green) gives way to pyroxene phases: clinopyroxene (CPX, purple) and orthopyroxene (OPX, orange). (b) Modulations in modal frequencies correlate with changes in cation concentration at the few percent level as determined by EDS. The spectroscopically distinct $300 \mathrm{~nm}$ wide transition band between CPX and OPX observed by nanoFTIR (OCPX, boxed in (a)) is attributed to an OPX / CPX superlattice verified by TEM (d, top), indicative of crystal shock. (d, bottom) HRTEM zoom onto the boxed region resolves the OPX / OCPX interface. (c) Electron diffraction from the respective pyroxene phases confirms an aperiodic lattice superstructure within this band.

\section{References:}

[1] D. Brownlee et al., Comet 81P/Wild 2 Under a Microscope. Science 314, 1711 (2006).

[2] F. Huth et al., Nano-FTIR Absorption Spectroscopy of Molecular Fingerprints at $20 \mathrm{~nm}$ Spatial Resolution. Nano Lett. 12, 3973 (2012/08/08, 2012).

[3] Z. Gainsforth et al., Constraints on the formation environment of two chondrule-like igneous particles from Comet 81P/Wild 2. Meteoritics \& Planetary science (in prep.), (2013).

[4] R. C. Ogliore et al., The Astrophysical Journal Letters 745, L19 (2012). 